\title{
Development of a Low Data Event Timer for Monitoring an Advancing Crack in Fracture
}

\section{J. Macon", P. D. Totman, M. L. Bodily, R. L. Everton, and M. R. Eggett Thiokol Inc., PO Box 707, M/S 243, Brigham City, UT 84302-0707}

\begin{abstract}
Monitoring the crack position and velocity in a fracture specimen can be difficult and laborious. In addition, the data storage requirements can be considerable depending upon the testing conditions. A low data event timer was developed to alleviate these problems. The test apparatus was applied to cantilever beams bonded with a structural epoxy and tested under different conditions such as stable to unstable transitions and different temperature extremes. The results indicate that the approach eliminates problems associated with other types of crack measurement and greatly simplifies the measuring process.
\end{abstract}

Key Words: A. Epoxy/epoxides; B. Fracture mechanics; D. Fracture; Crack location *Corresponding Author. Tel.: (435)-863-3035, Email: David.Macon@atk.com

C2004 ATK Thiokol Inc., an affiliate of Alliant Techsystems Inc. 


\section{INTRODUCTION}

One of the more common methods used in fracture mechanics analysis is the energy failure criterion. The energy approach assumes that fracture occurs when enough energy is available to exceed the materials resistance. For linear elastic materials, Irwin put this in terms of a critical energy release rate, $G_{c}$, with crack extension occurring when this value is exceeded [1]. In the case of a cracked plate of uniform width that is dead loaded, the critical energy release rate may be written as

$$
G_{C}=\frac{P_{C}^{2}}{2 b} \frac{\partial C}{\partial a}
$$

where $P_{C}$ is the critical load at which crack extension occurs, $b$ is the width of the plate, $C$ is the compliance of the specimen, and $a$ is the crack length.

For nonlinear elastic materials, Rice introduced a nonlinear critical energy release rate, $J$, also known as the J-integral [2]. The J-integral can be written in terms of load and displacement for a crack in a plate of uniform thickness as

$$
J=\int_{0}^{P}\left(\frac{\partial \delta}{\partial a}\right)_{P} d P \quad \text { Fixed load condition }
$$




$$
J=\int_{0}^{\delta}\left(\frac{\partial P}{\partial a}\right)_{\delta} d \delta \quad \text { Fixed displacement condition (3) }
$$

where $\delta$ is the displacement.

Experimental determination of these energy release rates requires knowledge of the load, displacement, and crack position (often as a function of time). Load and displacement measurements are generally determined through the use of load cells and extensometers, respectively. Knowledge of the crack location, or crack velocity in the case of dynamic fracture can be difficult to experimentally measure and is usually labor intensive. Generally, there are five approaches used for measuring crack location and velocity. These methods are optical, fractography, compliance, acoustic, and potentiometric [3].

Optical methods measure the location of the crack tip by observing the surface of the test specimen. For slow crack speeds, magnifying instruments used in conjunction with a measuring scale allow for accurate crack velocity measurements. As the crack velocity increases, high-speed recording devices may be required [4-5]. Special techniques such as photoelasticity, Moiré fringes, or shadowgraphy can improve the measurement accuracy $[3,6]$.

Fractography is a post-mortem examination method. The post-failure topography of the fracture surface can yield information about the crack's shape and position of the crack. One extension of this technique is to superimpose an ultrasonic shear vibration on the 
main load [3]. Such an approach allows a direct correlation between crack velocity and microscopic morphology [7].

The compliance method uses a constitutive relation to describe the crack position in terms of the specimen dimensions, modulus, and load. The accuracy of the relation can be improved by including a strain term, which can be explicitly measured using a strain gage. This technique is usually restricted to special geometries [3].

The acoustic method measures the propagation of a wave through a solid continuum. Regions where the material properties are changing will cause a change in the propagating wave. Artifacts such as a discontinuity will cause a reflection of the wave, which can be experimentally measured [3]. Such methods have been used to measure fracture in a pressure vessel [8].

One of the more common approaches and the one that will remain the topic of this paper is the potentiometric technique. This method measures the change in electrical conductivity of the test material or of gages attached to the specimen. For the former, the potential of the conducting material changes as a crack propagates through it. Through the use of a calibration curve, the crack position and velocity can be established [3]. The latter method measures the variation of electrical resistance of a conductive coating deposited on the crack path [9]. 
The conductive coating may be placed directly on the test specimen if the material is insulative or onto an insulating support. Generally, the attached gage comes in one of two forms; continuous [3,9-10] or grid [11-12]. An illustration of both grid types can be seen in Figure 1. The resistance grid gives discrete steps in a resistance versus time curve, but allows no measurements between each circuit. The continuous gage allows continuous measurements to be made, but has some non-linear behavior in a resistance versus time curve, and a calibration curve is required for each gage geometry.

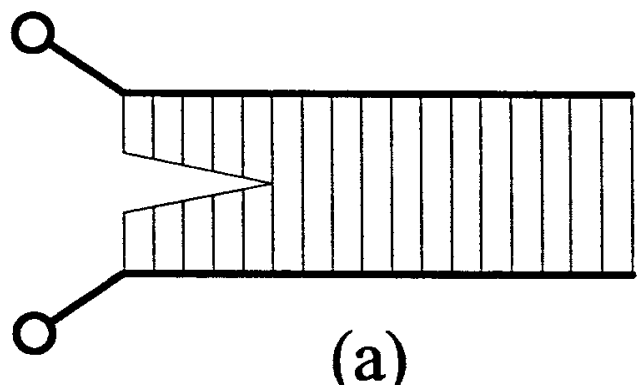

(a)

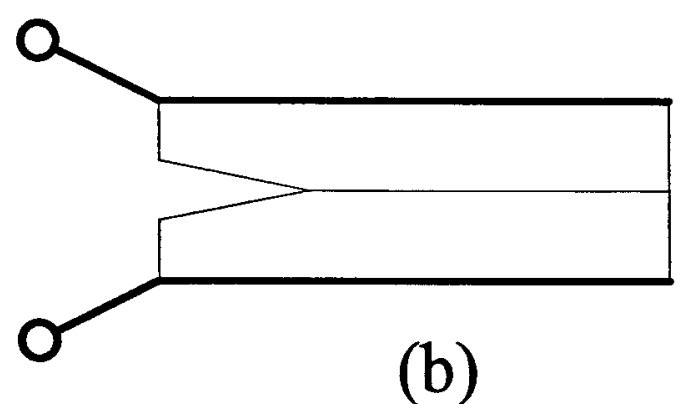

Figure 1: Conductive gages: (a) Grid. (b) Continuous.

The primary problem with the potentiometric gages is that the crack in the test material may not track the same as in the attached gage. Possible reasons include test material and insulating layer with different stress-strain behaviors, or conductive material that is too ductile or brittle. Another limitation of the potentiometric gage is that the gage does not track the explicit location of the crack tip. This can present problems if the fracture test requires something besides mode I opening at a single edge of the specimen (e.g., double edge notched tension panels as shown in Figure 2). 


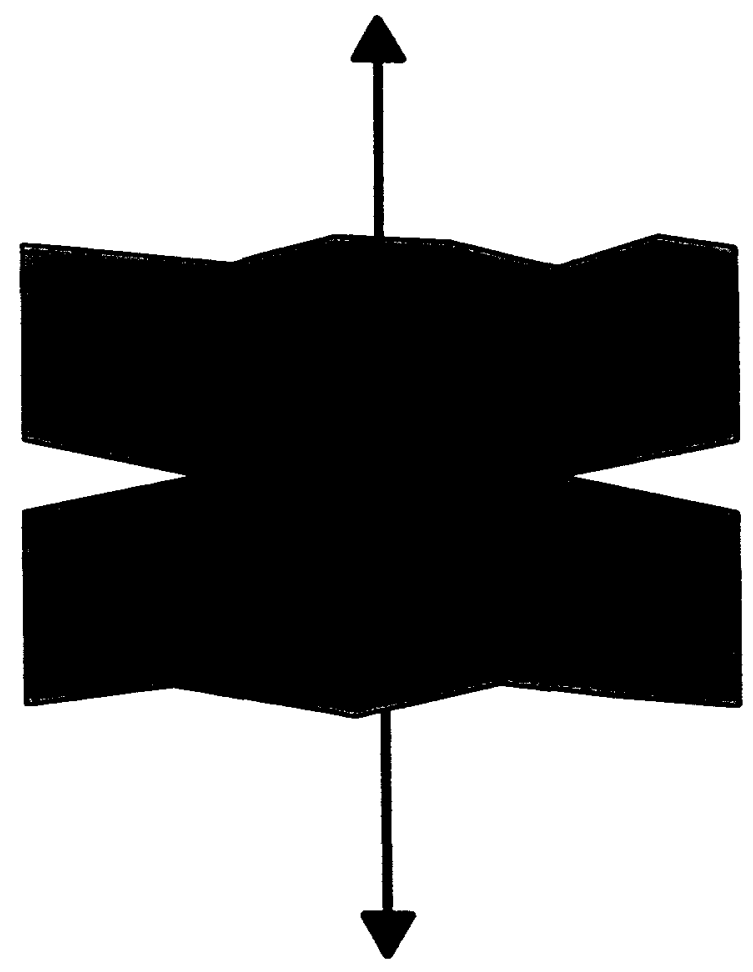

Figure 2: Schematic of a double edge delamination specimen.

One issue that applies to all of the listed testing techniques (i.e., optical, fractography, compliance, acoustic, or potentiometric) is that the test-sampling rate scales proportionally to the crack velocity. At very fast crack speeds, the sampling rate is often in the order of microseconds. The data file associated with such a failure event can be of large magnitude and significant data reduction may be required.

At ATK Thiokol, in excess of 2500 adhesive fracture tests of the tapered double cantilever beam (TDCB) variety are conducted annually. The test times vary from a few seconds to several days. Measuring the crack position and velocity of even a small percentage of these tests is difficult because of the data measurement/recording 
requirements. This challenge lead to the development of a Low Data Event Timer (LDET) to be used in conjunction with a crack propagation gage than can be easily attached to a double cantilever beam (DCB) geometry.

\section{Experimental}

\subsection{Low Data Event Timer}

Figure 3 illustrates the basic schematic of the LDET layout. There are three essential components:

1. Programmable logic device (PLD), such as a field programmable gate array, coupled with a high-resolution clock and a RAM storage device.

2. A means such as electrical, optical, or radio frequency for transferring a signal to the PLD.

3. A sensor array made up of individual, breakable elements, which for this setup is the crack propagation gage. Other possible elements include photoelectric, piezoelectric, and magnetic switches. 


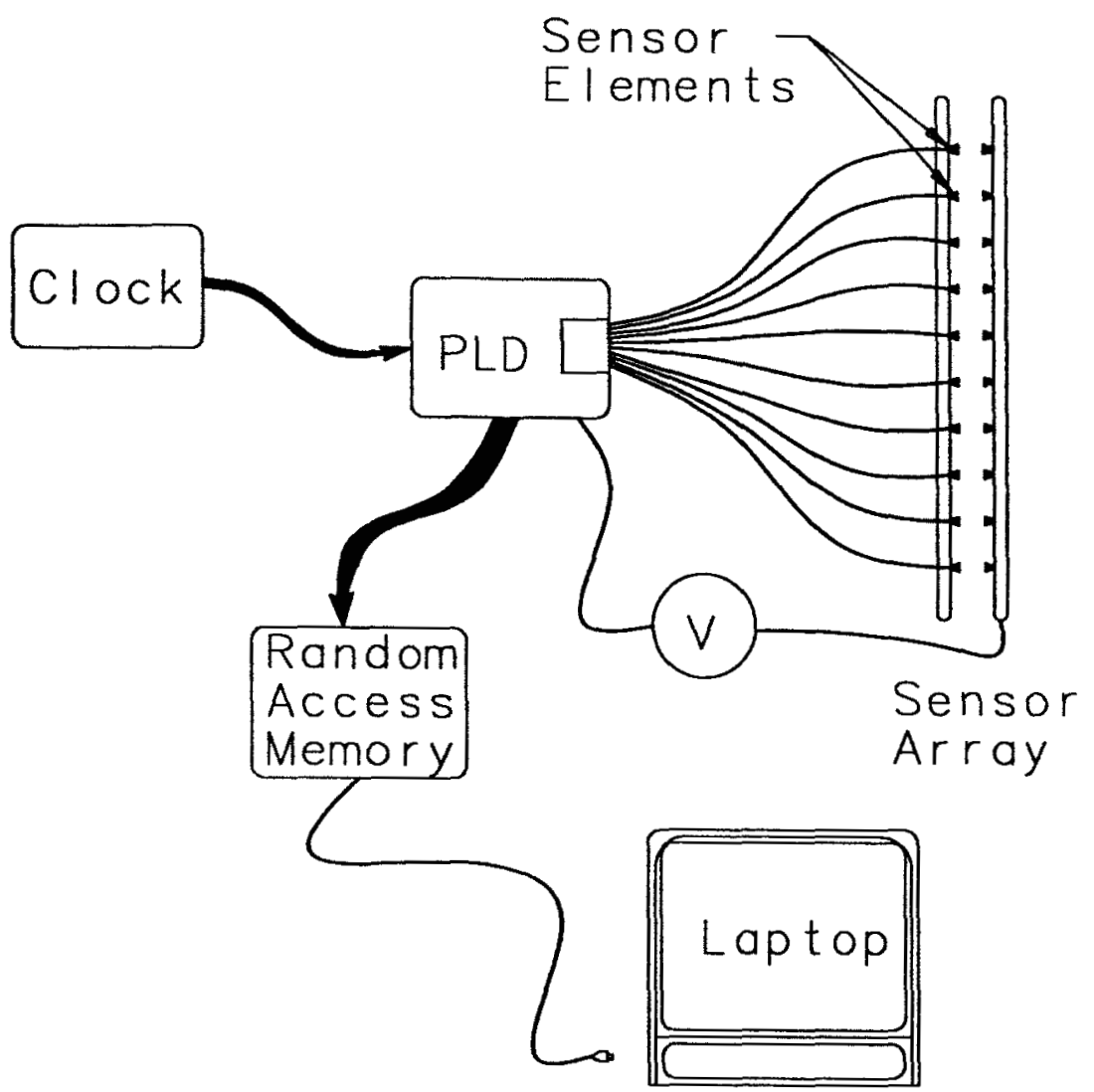

Figure 3: Schematic set up of low data event timer.

The PLD is programmed to detect a voltage change in individual electrical conductors.

Up to several hundred electrical conducting elements can be simultaneously monitored by the device. A memory location inside the PLD is assigned to each element. When the circuit voltage changes, the clock time (time stamp) from the chip is recorded and stored in the elements memory location. The time stamp for all elements is then downloaded to a computer after the event. 


\subsection{Crack Propagation Gage}

Figure 4 illustrates one possible schematic for a crack propagation gage. It is a flexible printed circuit that is designed to be bonded to a variety of DCB test specimens. There is a localized region on the gage (tear region) that is designed to tear as a crack propagates through the adhesive bondline.

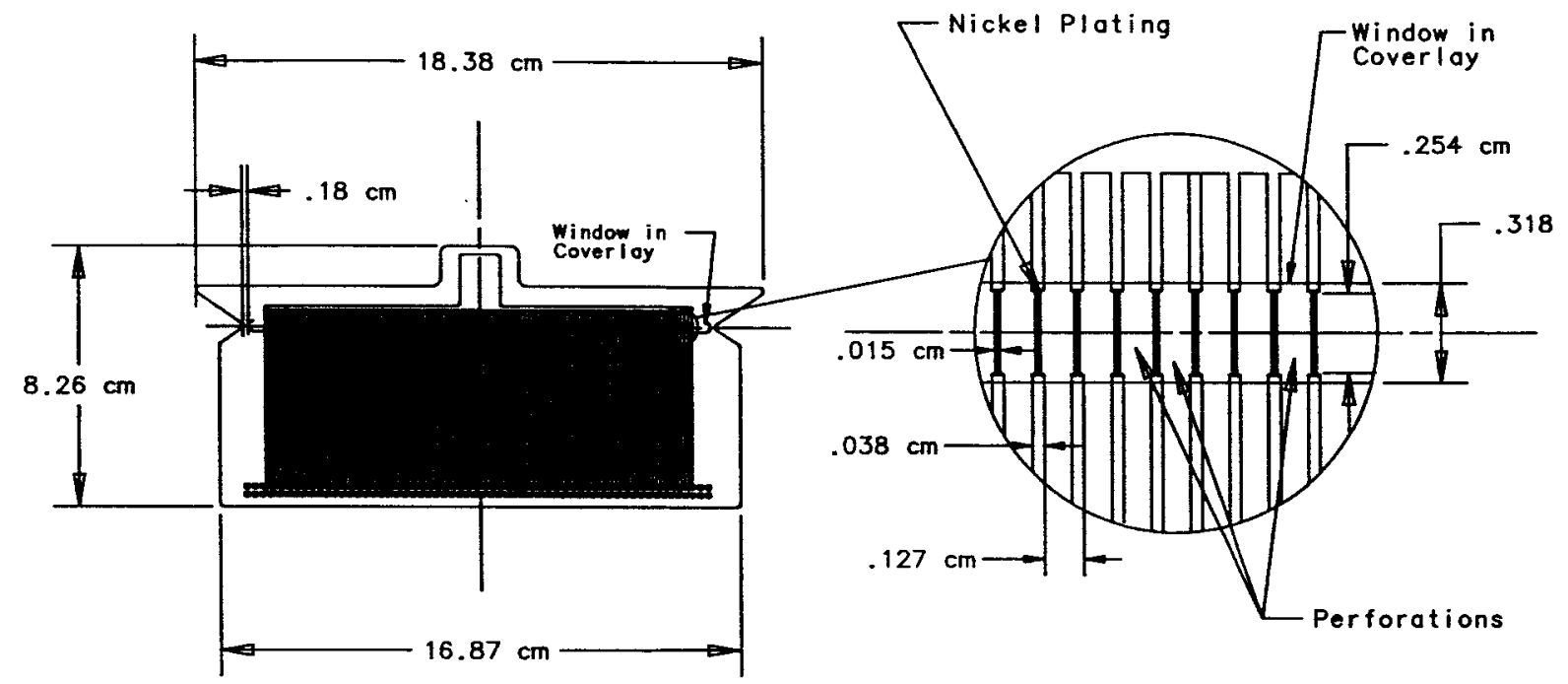

Figure 4: Crack propagation gage schematic.

Gages are manufactured by removing copper from $0.00254 \mathrm{~cm}$ thick; copper clad Kapton ${ }^{\otimes}$ sheets to form individual electrically conductive elements at specified distances. The element distance is $0.127 \mathrm{~cm}$, but this spacing can be changed if a different resolution is required. The elements are $0.038 \mathrm{~cm}$ wide, and neck down to $0.015 \mathrm{~cm}$ wide in the tear region. The length of the necking region in the elements is $0.254 \mathrm{~cm}$. 
The tear region is $0.318 \mathrm{~cm}$ wide and runs the length of the conductive elements. All Kapton $^{\circledR}$ is removed between the elements in the tear region. The necked down elements are nickel-plated to make the copper brittle in this area. After nickel plating, a 0.00254 $\mathrm{cm}$ thick Kapton ${ }^{2}$ cover lay is applied to the gage in all areas but the tear region. The nickel-plated elements in the tear region are left bare. The gage is electrically connected to the PLD through the use of two 60- pin, through-hole male connectors. The through holes are nickel-plated and fiberglass reinforced.

A common bus on one side of the tear region connects the conductive electrical elements. The other end of the element is routed to the PLD. When an element is broken in the tear region, current will stop flowing in that element. The presence of current flowing in the elements is monitored inside the PLD. When current loss is detected in an element, the time is stored in the memory register for that element.

\subsection{DCB Preparation and Testing}

The crack measurement apparatus was tested using cantilever beams of the tapered variety. The beams were made of D6AC steel and conformed to ASTM D 3433. A schematic of the specimen is shown in Figure 5. 


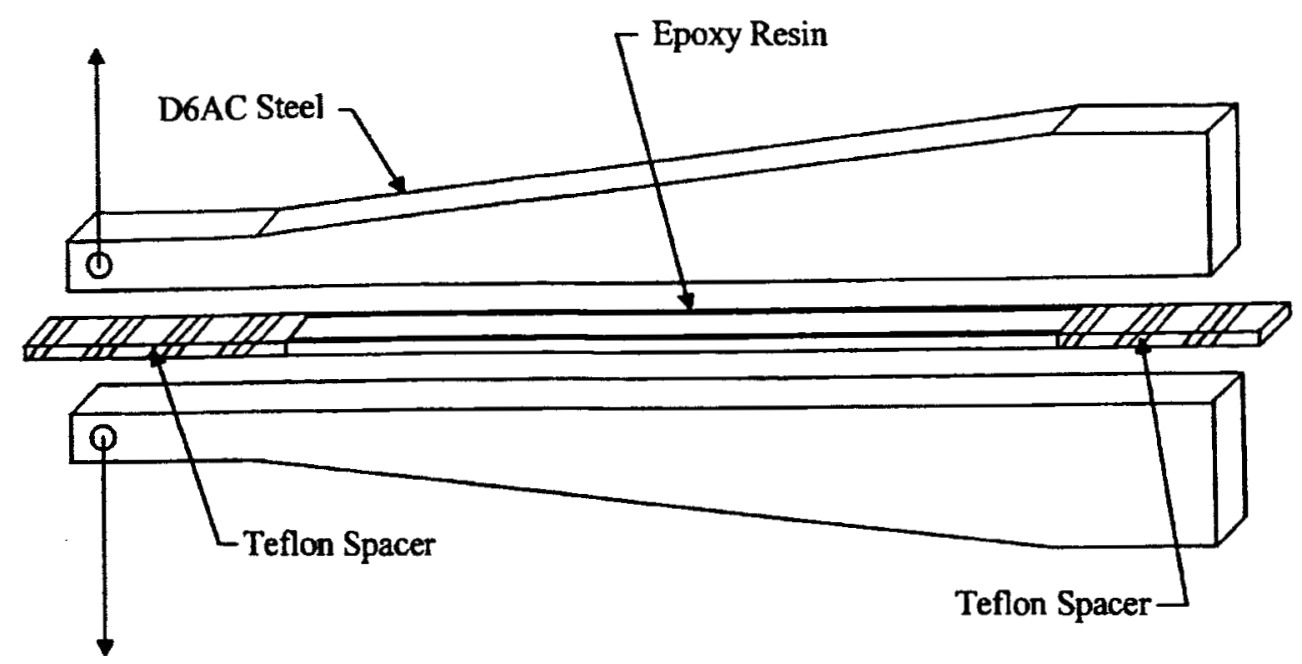

Figure 5: Geometry of the tapered double cantilever beam specimens.

The TDCB adherends were bonded using a thixotropic structural epoxy, TIGA $321^{\circledR}$, manufactured by Resin Technology Group (RTG). Excess adhesive was applied to each beam and allowed to extrude out as the two adherends were pressed together. The extruded adhesive was removed from the side of the beams prior to curing. The bondline thickness was maintained at $0.127 \mathrm{~cm}$ using Teflon $^{\circledR}$ spacers controlled to a $10 \%$ dimensional tolerance. After bonding, the specimens were placed in a jig that helped maintain alignment and a slight compressive load was applied. After an elevated temperature cure, the specimens were allowed to equilibrate at $22^{\circ} \mathrm{C}$.

The crack propagation gages were applied to the adherends using TIGA $321^{\circledR}$. In the tear region of the gage, adhesive was allowed to fully encapsulate the exposed conductive elements. This helped ensure that the crack propagation gage failed at the same rate as the TDCB specimen. After bonding the gages, the adhesive was cured at the same elevated temperature. 
The bonded adherends were tested using a Satec ${ }^{\circledR}$ Unidrive. The tests were performed in displacement control at a variety of crosshead speeds. The different speeds were chosen to give both stable and unstable failure modes. An environmental chamber was used in conjunction with the Unidrive when temperature testing was performed.

\section{Results and Discussion}

Experiments were conducted to evaluate the viability of the LDET system under two different test conditions.

1. Stable versus Unstable Crack Propagation - Stable crack propagation is the most common behavior observed with this adhesive under standard test conditions, but the transition to unstable propagation is often instantaneous and unexpected. The LDET system is designed to easily monitor the crack location during these types of transition.

2. Temperature Effects - Measuring crack locations at different test temperatures can present certain challenges. The use of environmental chambers can prevent easy access to the test specimens. Test methods that require complicated equipment (e.g., optical recording devices, acoustic methods, etc.) may have problems when working through an environmental chamber. Potentiometric techniques such as the continuous conductive gage may have issues with the temperature changes involved. Since the resistance of a material changes with temperature, calibration 
curves would be required for each test temperature. The LDET device avoids these problems and only requires a means for transferring a signal from the sensor array to the PLD, such as a wire passed through the environment chamber.

One application where the LDET system should be readily applicable is static loading. This is the process where fracture occurs some time after the application of a constant load. The time scale can also be quite long with failure occurring in months instead of minutes. The LDET method should be well suited to this task. Instead of constantly monitoring the status of the gage, a time-stamp would be generated whenever the status of the gage changes. This favorably lends itself to the longer time intervals.

\subsection{Stable versus Unstable Crack Propagation Results}

The transition from slow to unstable crack growth represents a significant change in crack velocity. Stable cracks travel through TIGA $321^{\circledR}$ at around $1 \times 10^{-4} \mathrm{~m} / \mathrm{s}$ and then can suddenly accelerate to velocities of approximately $410 \mathrm{~m} / \mathrm{s}$ as the failure becomes unstable. The data logging requirements for such a dramatic change in velocity is considerable. To adequately acquire information about the catastrophic failure event, sample rates of up to $10 \mathrm{MHz}$ are required, and the associated data storage requirements are also significant. The LDET apparatus circumvents this issue by generating a timestamp only when an element is broken by the advancing crack. Otherwise, the PLD continues to monitor the integrity of the circuit and waits for a trigger that will tell it to 
record another time stamp with a clock that has a resolution of $\sim 12 \mathrm{MHz}$. No additional data are generated.

Figure 6 illustrates a typical plot of load and crack position versus time using a TDCB adherend outfitted with a crack propagation gage tested at ambient conditions. The load is given on the left abscissa with the crack position on the right abscissa. Inspection of Figure 6 shows the load trace increasing in magnitude until crack initiation starts. The crack continues to grow with increasing load ( $R$-curve behavior) until a stable steady state is reached. The crack continues to grow in a stable manner until catastrophic failure occurs down the length of the beam. 


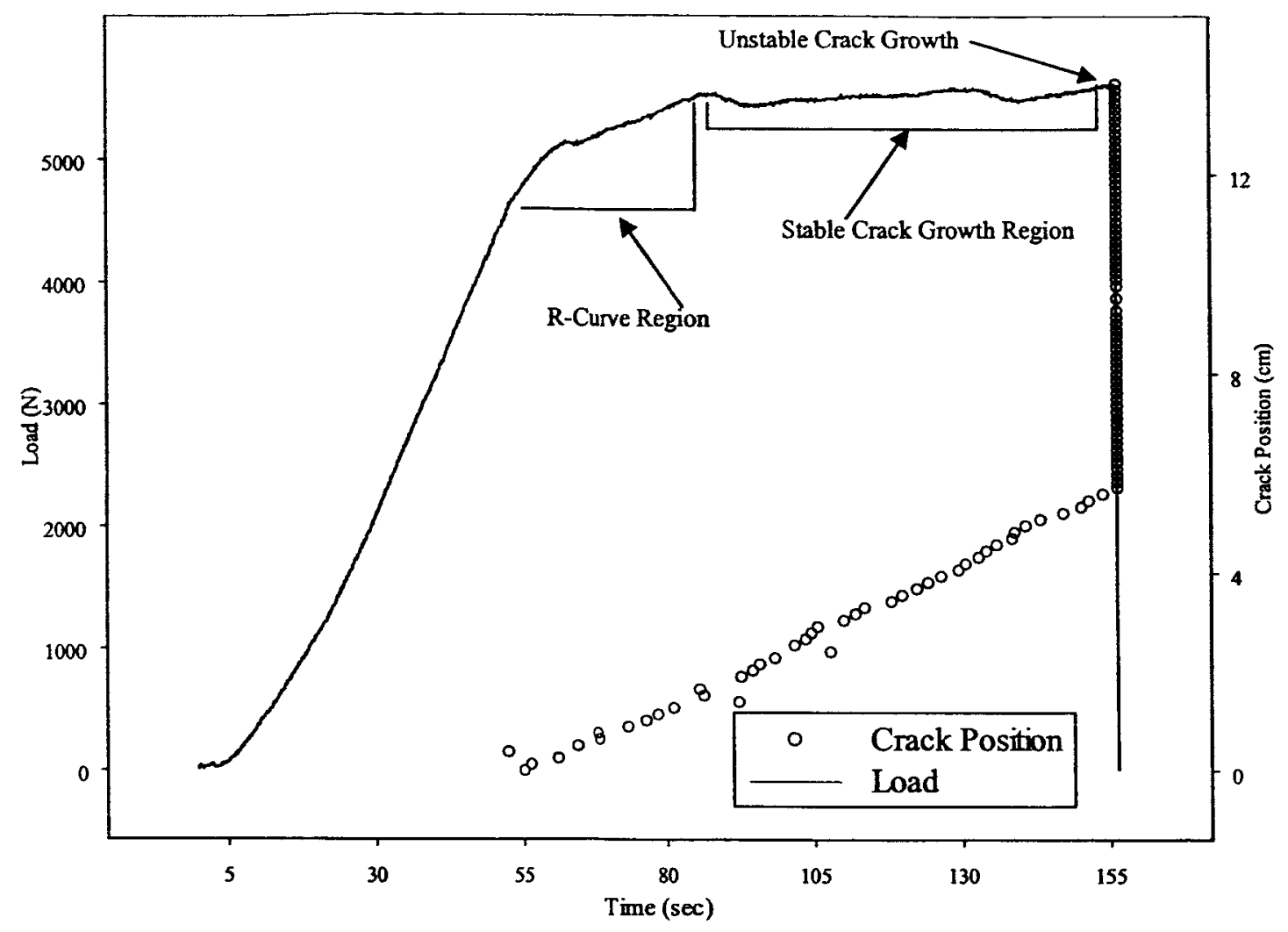

Figure 6: Plot of load and crack position versus time for a TDCB specimen tested at $22^{\circ} \mathrm{C}$.

The critical mode I fracture energy, $G_{I C}$, for a cantilever beam is

$$
G_{I C}=\frac{P_{C}^{2}}{2 b} \frac{\partial C}{\partial a}
$$

where $P_{C}$ is the critical load, $b$ is the width of the beam, $C$ is the compliance of the adherend, and $a$ is the crack position. This relation assumes linear elastic behavior. Mostovoy et al. used simple shear-corrected beam theory to express the fracture energy for adhesively bonded DCB adherends as 


$$
G_{I C}=\frac{P_{C}^{2}}{2 E b^{2}}\left(\frac{3 a^{2}}{h^{3}}+\frac{1}{h}\right)
$$

where $E$ is Young's modulus of the beams, and $h$ is the beam height [13]. The TDCB specimen is designed so that $\partial C / \partial a$ is a constant. This was done by machining the TDCB adherend so that the term in brackets is constant i.e.,

$$
\left(\frac{3 a^{2}}{h^{3}}+\frac{1}{h}\right)=m
$$

where $m$ is a shape factor. For ASTM D 3433, $m$ is set equal to $35.43 \mathrm{~cm}^{-1}$.

The advantage of this geometry is that the crack grows linearly as the adherend is loaded at a constant displacement rate. This behavior is observed in Figure 6 where the crack grows linearly with time (constant displacement rate). The gage accurately measured the crack position through both the stable and unstable failure events. The element breakage times ranged from $4.1 \mu$ s to $2.1 \mathrm{~s}$. The LDET system easily measured this sixth-order change in magnitude.

Figure 7 highlights the unstable crack growth resulting from catastrophic failure as shown in Figure 6. The crack position and time are rescaled so that zero on both scales occurs at the instant of unstable crack growth. It can be seen that the catastrophic event is over within 250 microseconds. There is some scatter in data with some of the elements breaking out of order. The cause of the elements breaking out of order is uncertain but is 
most likely caused by electrical cross-talk between adjacent elements. Loss of current in one element can induce voltages large enough to cause a sympathetic trigger in an adjacent element.

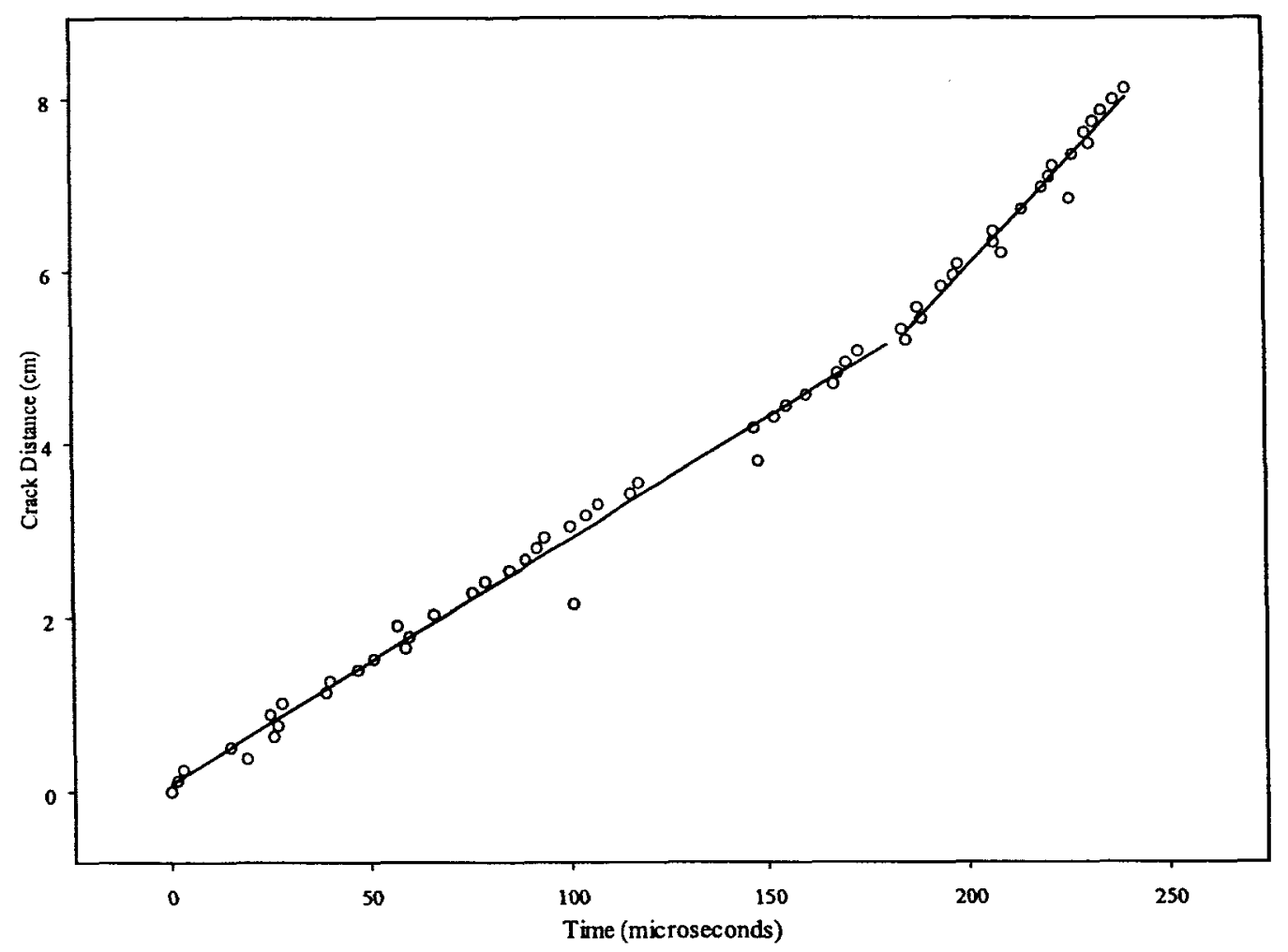

Figure 7: Plot of crack position versus time for the catastrophic failure event show in Figure 6

The crack velocity is constant until a certain point at which the crack begins to accelerate. It is believed that this phenomenon is caused by the advancing crack meeting reflected waves. These waves are generated during the failure event and travel down the length of the beam until a free surface is reached. The reflected waves return to the point of initiation and collide with the advancing crack tip. The velocity in the initial linear 
region is $286 \mathrm{~m} / \mathrm{s}$. It was previously reported that the measured speed of sound in TIGA $321^{\circledR}$ is $2794 \mathrm{~m} / \mathrm{s}$ [14]. So the reflected wave could easily reach the end of the specimen and return in time to meet the advancing crack.

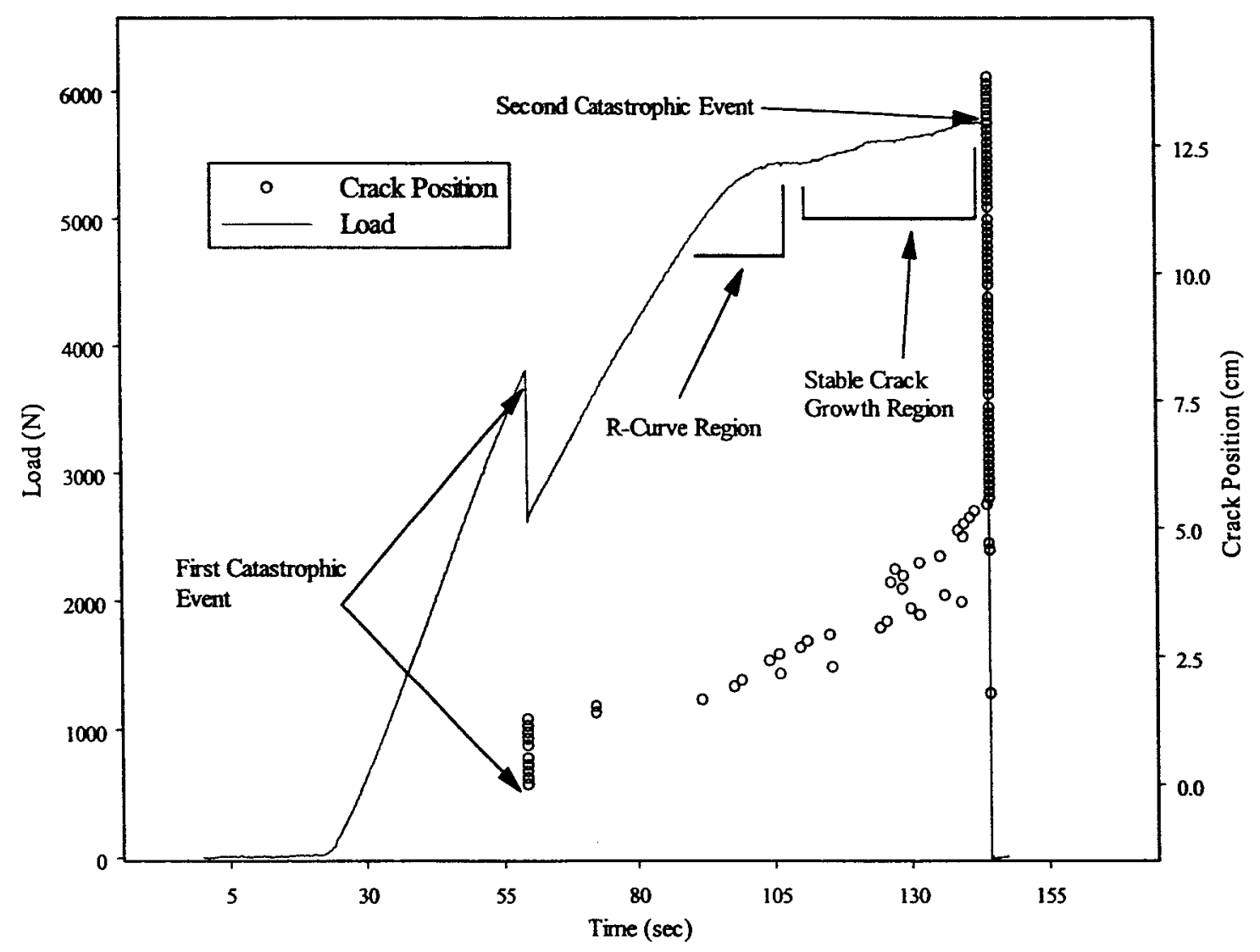

Figure 8: Load and crack location versus time for a mixed stable/unstable failure event.

At higher deformation rates, the failure shifts from stable crack growth to a mix of stable and unstable failure modes. A typical test result is shown in Figure 8 where load and crack location is plotted versus time for a TDCB sample tested at room temperature but at a faster crosshead speed. The specimen loads until the initial catastrophic failure event happens, which is followed by reloading of the specimen until stable crack growth 
occurs. Finally when the material is no longer able to dissipate the input energy, catastrophic failure occurs down the remaining length of the beam.

The crack propagation gage was able to accurately catch the initial failure event. During the reloading period, a few circuits broke out of order at around 72 seconds. The gage started recording crack propagation again in the R-curve region and on into the stable crack growth region. There is however considerable data scatter in these regions, as the elements break out of order. This trend was observed for most beams that failed with mixed stable/unstable behavior. It is speculated that small power surges caused by the collapse of an electrical field created by current loss in a broken element could be causing false triggers in adjacent elements. Though there is scatter in the stable crack growth region, the gage is still able to measure the two catastrophic events. These regions were plotted separately in Figure 9. Inspection of the figure shows the high accuracy to which the crack propagation gage can monitor the events. Crack velocities of $215 \mathrm{~m} / \mathrm{s}$ and 408 $\mathrm{m} / \mathrm{s}$ were measured for the first and second failure events, respectively. 

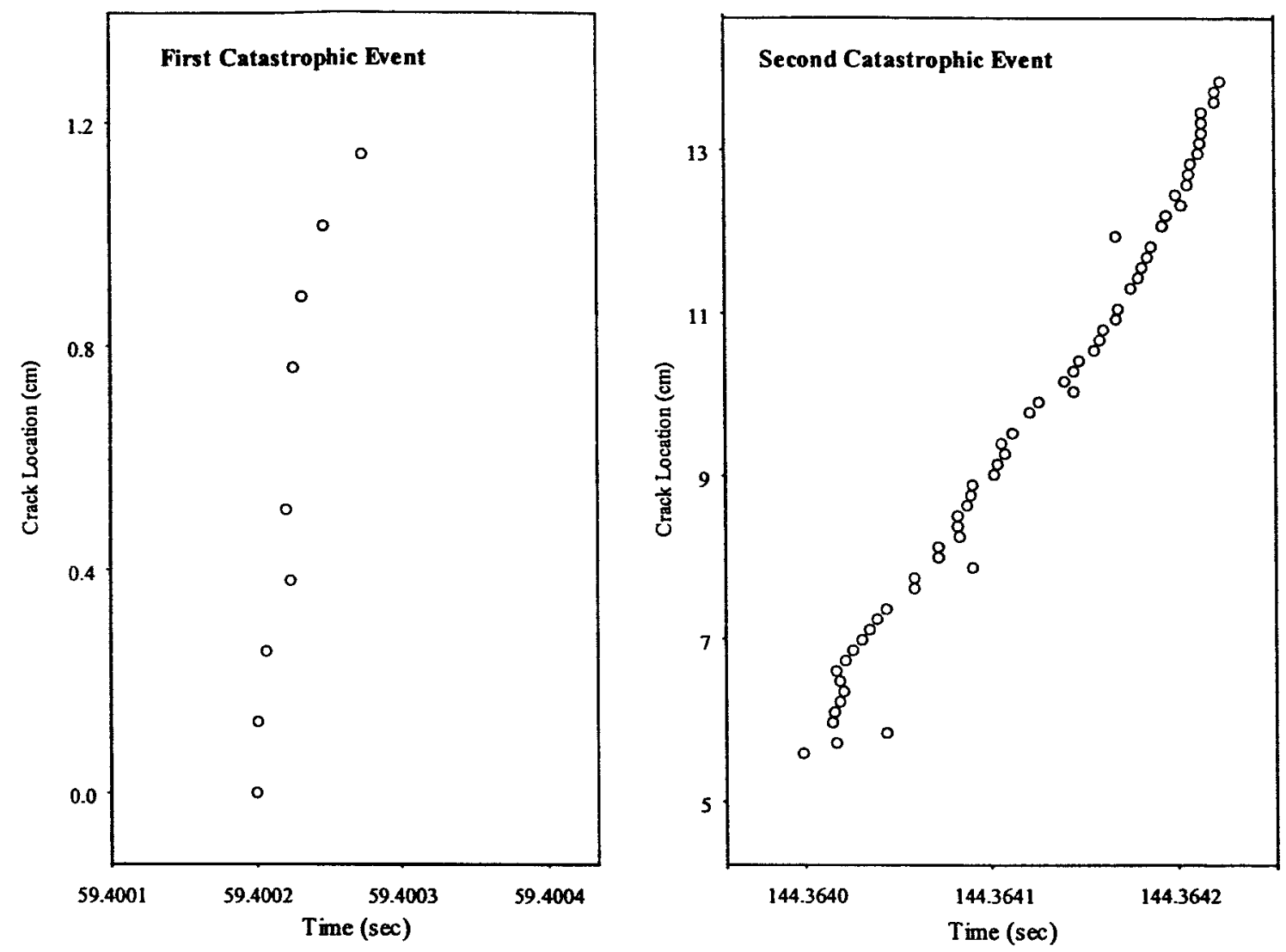

Figure 9: Plot of load versus time for the two catastrophic failure events shown in Figure 8.

\subsection{Temperature Testing Results}

The capability of the LDET apparatus to record crack growth data at a variety of test temperatures was evaluated by testing TDCB adherends at $-28.9^{\circ} \mathrm{C}$ and $46.1^{\circ} \mathrm{C}$. TIGA $321^{\circledR}$ has very different material properties at these two test temperatures. At $-28.9^{\circ} \mathrm{C}$, the adhesive acts as a glassy material with no yield point, and fracture is brittle with the crack advancing through the bulk of the material. As the temperature approaches $46.1^{\circ} \mathrm{C}$, TIGA $321^{\circledast}$ softens as the glass transition temperature $\left(\sim 51.7^{\circ} \mathrm{C}\right)$ is approached. The 
failure transitions from cohesive within the adhesive to interfacial failure between the adhesive and the steel substrate as the test temperature increases.

Testing was conducted by placing the TDCB adherend and the load fixtures into an environmental chamber. The whole apparatus was conditioned at the relevant test temperature for a minimum of two hours. A data acquisition cable was run from the crack propagation gage, through the environment chamber, and then to the field programmable gate array.

\subsubsection{Cold Temperature Testing}

The load and crack advancement versus time plot for a TDCB adherend tested at $-28.9^{\circ} \mathrm{C}$ is shown in Figure 10. The load and crack position are shown on the Y-coordinates, and time is shown on the X-coordinate. The load is characterized by two catastrophic events. There is no R-curve or stable crack growth region as the TIGA $321^{\circledR}$ adhesive has no measurable yield point at $-28.9^{\circ} \mathrm{C}$ 


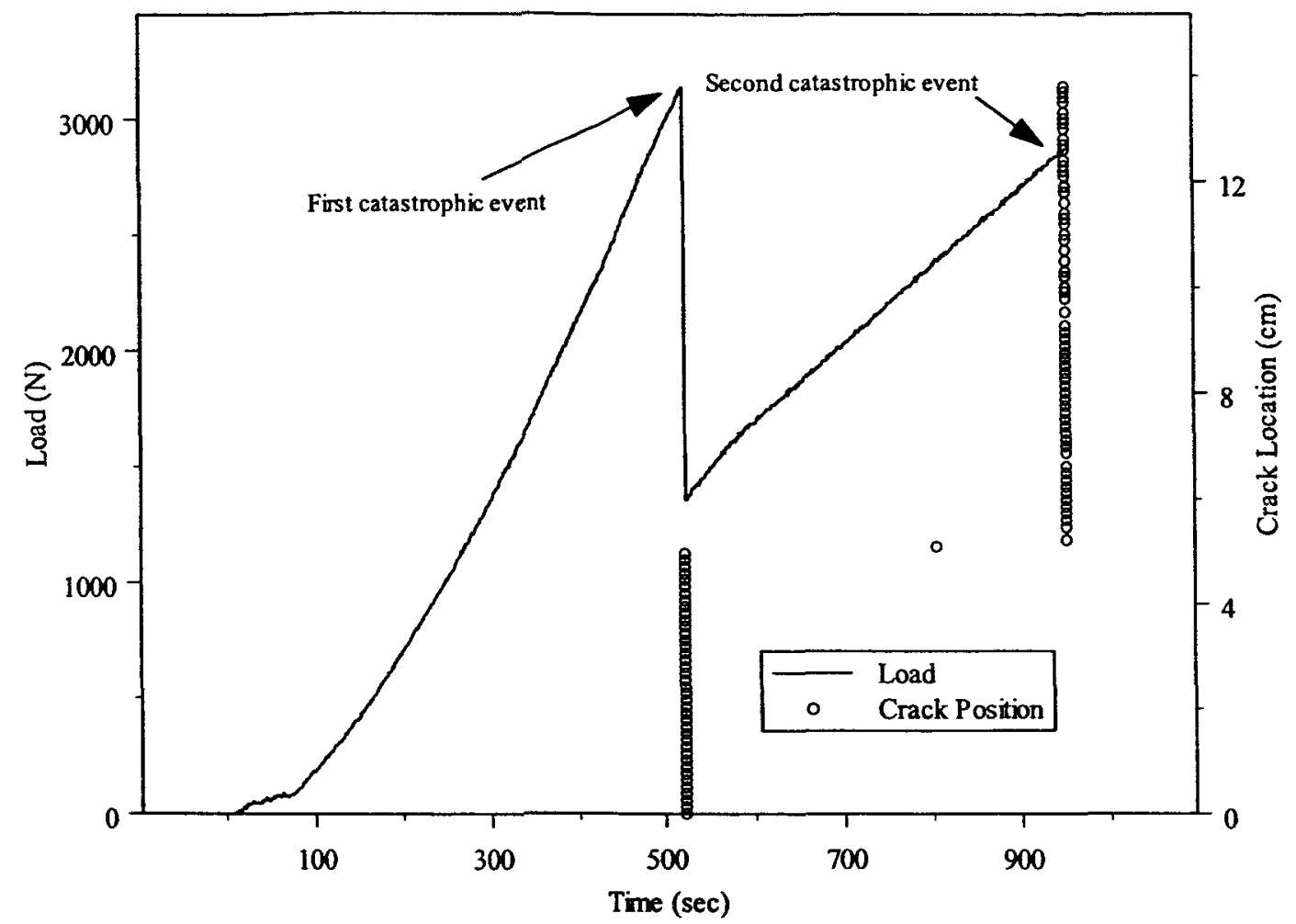

Figure 10: Load and crack location versus time for unstable fracture occurring at $-28.9^{\circ} \mathrm{C}$.

The crack position versus time profile for the two evens is illustrated in Figure 11. The crack velocity for the first catastrophic event deviates from linearity as the crack decelerates. The crack velocity for the initial linear section is $347 \mathrm{~m} / \mathrm{s}$. For the second catastrophic event, the crack velocity is $349 \mathrm{~m} / \mathrm{s}$. 

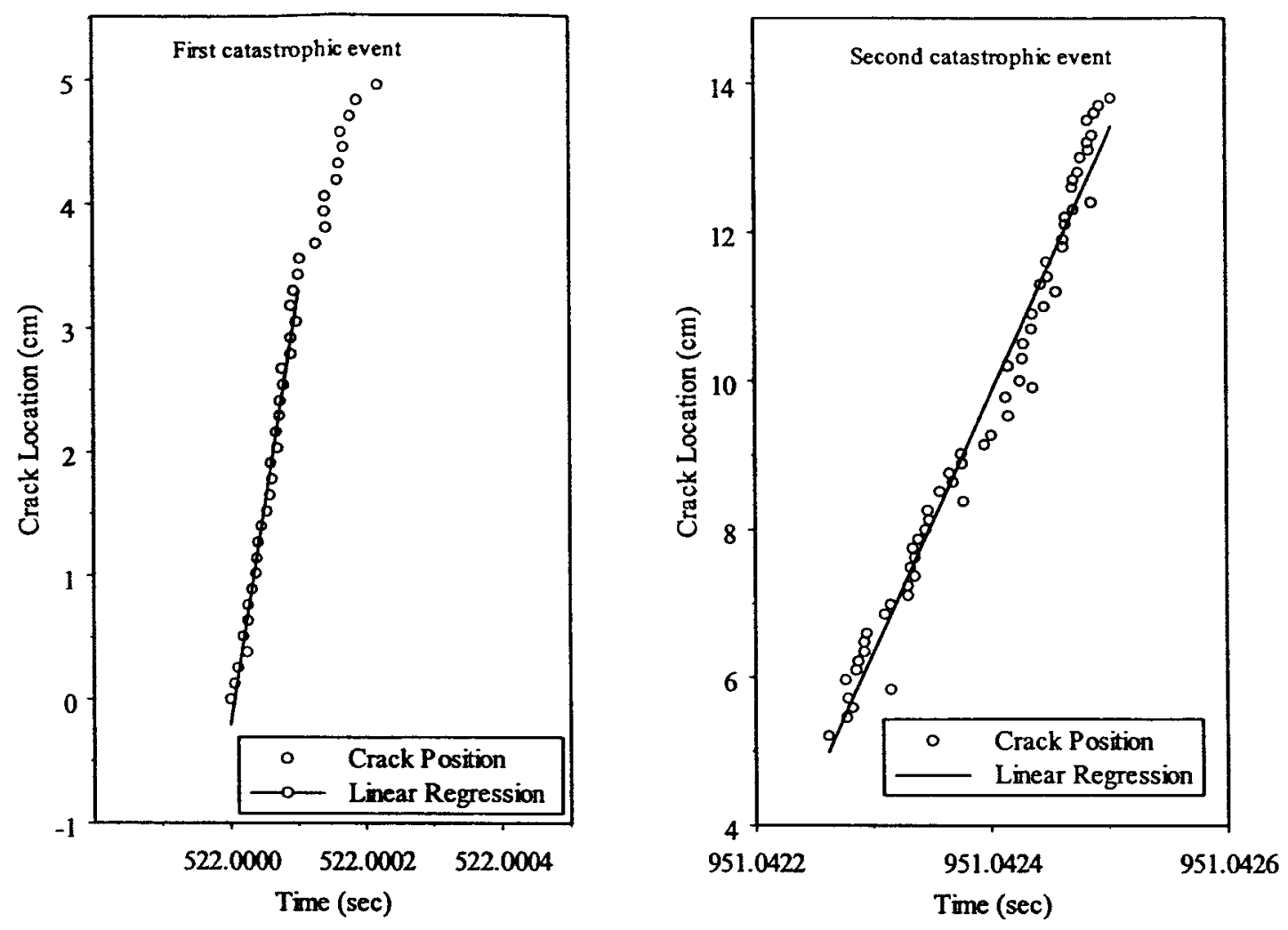

Figure 11: Plot of crack location versus time for the two catastrophic events shown in Figure 10.

\subsubsection{Elevated Temperature Testing}

The load and crack advancement versus time plot for a TDCB adherend tested at $46.1^{\circ} \mathrm{C}$ is shown in Figure 12. At this test temperature, Young's modulus for TIGA $321^{\circledR}$ shifts from $3.3 \mathrm{GPa}$ when tested at $22^{\circ} \mathrm{C}$ and a crosshead displacement speed of $5.08 \mathrm{~cm} / \mathrm{min}$ to $1.9 \mathrm{GPa}$ for an identical crosshead speed. 


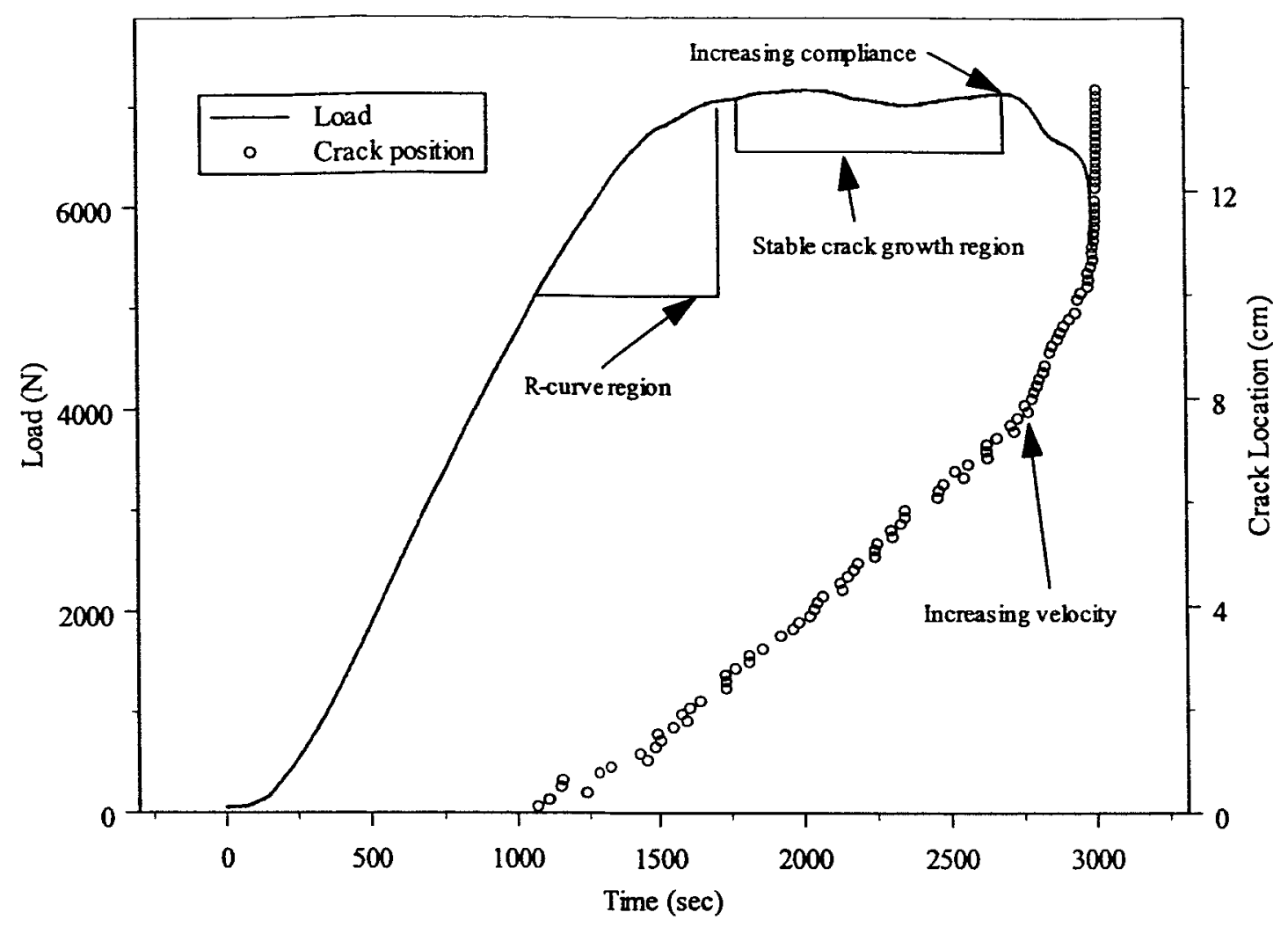

Figure 12: Load and crack location versus time for stable fracture occurring at $46.1^{\circ} \mathrm{C}$.

Figure 12 shows similar crack growth behavior to that observed when TIGA 321 is tested at $22^{\circ} \mathrm{C}$ (Figure 6). The R-curve region is larger in Figure 11 as would be expected as the material softens. Stable crack growth occurs at larger loads $(\sim 7000 \mathrm{~N}$ versus $\sim 5500 \mathrm{~N})$. One significant difference is the sudden increase in compliance in the load trace with an associated increase in crack velocity.

Testing at $-28.9^{\circ} \mathrm{C}$ and $46.1^{\circ} \mathrm{C}$ did not present any difficulty for the LDET apparatus. The setup was instantly adapted to the environmental chamber without further modification. The device can be used to test greater temperature extremes than those reported here. 


\section{Summary}

The LDET approach significantly simplifies the monitoring of the crack position and velocity in a DCB fracture specimen. A time stamp is generated only in the event of a broken circuit on the crack propagation gage. As a result, the total number of data points equals the number of broken circuits and a continuous data record is not required. This greatly reduces the data logging requirements. This is particularly useful when the testing condition can last for days, but the failure event can occur in microseconds.

The apparatus is easily adapted to temperature testing. A single cord was required to attach the crack propagation gage to the programmable logic device. No other modifications or correction (such as a new calibration curve) were required. The LDET apparatus is readily adapted to other fracture tests such as static loading. 


\section{References}

[1] Irwin GR. Sagamore Research Conference Proceedings 1956;2:289-305.

[2] Rice JR. J App Mech 1968;35:379-386.

[3] Stalder B, Béguelin P, Roulin-Moloney AC, Kausch HH. J Mat Sci $1989 ; 24: 2262-2274$.

[4] Gamby D, Delauménie V. Composites Part A 1997;28A:875-881.

[5] Dear JP. Polym Eng \& Sci 1996;36(9):1210-5.

[6] Takahashi K, Kido M, Arakawa K. Int J Fracture 1998;90:119-131.

[7] Sheng JS, Zhao YP. Int J Fracture 1999;98:L9-L14.

[8] Jingu T, Nezu K. Int J Pres Ves \& Piping 1990;42:379-389.

[9] Cudré-Mauroux N, Kausch HH, Cantwell WJ, Roulin-Moloney AC. Int J Fracture 1991;50:67-77.

[10] Béguelin P, Stalder B, Kausch HH. Int J of Fracture 1983;23:R7-R10.

[11] Sahraoui S, Lataillade J, Pouyet J. Exp Tech 1986;10(6):23-5.

[12] Fond C, Schirrer R. J Phys IV France 1997;C3(7):969-974.

[13] Mostovoy S, Crosley PB, Ripling EJ. J Mater 1967;2(3):661-681.

[14] Macon DJ, Anderson GL. J Appl Polym Sci 2002;86(8):1821-8. 